\title{
Fixation durations before word skipping in reading
}

\author{
REINHOLD KLIEGL and RALF ENGBERT \\ University of Potsdam, Potsdam, Germany
}

\begin{abstract}
We resolve a controversy about reading fixations before word-skipping saccades which were reported as longer or shorter than control fixations in earlier studies. Our statistics are based on resampling of matched sets of fixations before skipped and nonskipped words, drawn from a database of 121,321 single fixations contributed by 230 readers of the Potsdam sentence corpus. Matched fixations originated from single-fixation forward-reading patterns and were equated for their positions within words. Fixations before skipped words were shorter before short or high-frequency words and longer before long or low-frequency words in comparison with control fixations. Reasons for inconsistencies in past research and implications for computational models are discussed.
\end{abstract}

Eye movement control during reading is determined by both perceptual and language processes. Engbert, Longtin, and Kliegl (2002) distinguished three broad categories of models: sequential attention shift (SAS) models, guidance by attentional gradients (GAG) models, and primary oculomotor control (POC) models. These types of models differ in how they weigh the factors for which they are named and in how they conceptualize or implement their interactions (see Reichle, Rayner, \& Pollatsek, 2003, for a comparison of the models). All three types of models predict that short, high-frequency, and highly predictable words are skipped more often than are long words, low-frequency words, and words with low predictability, and this prediction is solidly established by experimental research (see Brysbaert \& Vitu, 1998, for a meta-analysis). A distinguishing feature of these models, however, concerns their predictions for fixation durations before these skipping saccades.

In principle, there are two different processes that predict increased fixation durations before skipped words: saccade cancelation and parafoveal preprocessing. By definition, in SAS models such as E-Z Reader the next word is the default saccade target. Skipping usually requires a cancelation of the default saccade program and the start of a new one targeting word $n+2$ (see, e.g., Engbert \& Kliegl, 2001; Reichle, Pollatsek, Fisher, \& Rayner, 1998). Therefore, "costs of skipping" were predicted and obtained in simulations (Reichle et al., 2003, Table 1). Moreover, the predicted cancelation costs are likely to decrease with an increase in the frequency of the skipped

This research was supported by Grants KL 955/3 and KL 955/6 from the Deutsche Forschungsgemeinschaft. We thank Petra Grüttner for research assistance and Marc Brysbaert, George McConkie, Antje Nuthmann, Alexander Pollatsek, Erik Reichle, and Martin Rolfs for their comments. Correspondence concerning this article should be addressed to R. Kliegl, Department of Psychology, University of Potsdam, P.O. Box 6015 53, Potsdam 14451, Germany (e-mail: kliegl@rz.uni-potsdam.de). word because cancelation usually occurs earlier for highfrequency words. Thus, in SAS models longer fixations before skipping are the consequence of saccade cancelation, and cancelation costs should be inversely related to the frequency of the skipped word.

Alternatively, if we allow parallel processing of words within the perceptual span, as GAG models do, longer fixations imply a longer accumulation of parafoveal information. This will increase the probability of complete lexical access and, consequently, of skipping word $n+1$ (see, e.g., Engbert et al., 2002; Reilly \& Radach, 2003). Therefore, the second reason for skipping might be longer parafoveal processing during the last fixation. Thus, in GAG models long fixations are a cause, not a consequence, of skipping. Note, however, that the link between fixation durations and subsequent skipping is much less tight here than in the case of SAS models, especially for models such as SWIFT (Engbert et al., 2002), where saccade programs are mostly started autonomously.

Finally, POC models have traditionally assumed that saccades are generated from a distribution of saccade amplitudes that may be adjusted to the difficulty of the current text or text segment (McConkie, Kerr, \& Dyre, 1994). In the context of skipping, these theories were concerned primarily with effects on saccade amplitudes; they did not predict a strong modulation of fixation durations before skipping, assuming that the next saccade target is determined very early in the fixation, leading to a no-relation hypothesis (Radach \& Heller, 2000). Thus, rather than any consistent effect, an inconsistent effect of skipping on the prior fixation duration would be claimed in support of these models.

Even this rough sketch of theoretical predictions about fixation durations before skipped words underlines their importance for model evaluations (Kliegl \& Engbert, 2003; Radach \& Heller, 2000). Empirically, fixations before skipped words range from $84 \mathrm{msec}$ longer to $26 \mathrm{msec}$ shorter than those before nonskipped words. The three em- 
pirical studies cited by Reichle et al. (1998) in support of longer fixations before skipped words reported increases of +3 to $+7 \mathrm{msec}$ (Hogaboam, 1983, Tables 18.1 and 18.3), +21 msec (Pollatsek, Rayner, \& Balota, 1986, p. 126), and $+38 \mathrm{msec}$ (Reichle et al., 1998, p. 147). In addition, Pynte, Kennedy, and Ducrot (2004, Table 3) found that longer fixations before the short French prepositions $d e$ and $d u$ were skipped in four conditions (i.e., $+17,+84$, +42 , and $+60 \mathrm{msec}$ ). In the same journal issue, however, Drieghe, Brysbaert, Desmet, and De Baecke (2004, Table 7) reported (mostly) shorter fixations for combinations of predictability and word length (i.e., $+3,+9,-26,-13$, -11 , and $-11 \mathrm{msec}$ ). Similarly, Radach and Heller (2000, Table 2) reported skipping effects of $-16,+2$, and $-26 \mathrm{msec}$ for fixations on eight- to nine-letter-long words before words with three, four, and five or more letters, respectively. Finally, in analyses of large corpora, the effects were statistically not reliable or tended to be negative (see, e.g., McConkie et al., 1994; Radach \& Heller, 2000). Obviously, this experimental variance needs to be explained.

The diversity of earlier results could be due to their correlational nature (in that skipping is not under experimental control) combined with a less-than-optimal selection of fixations for statistical analysis. For example, within texts there may be easy segments that induce a tendency toward skips and short fixations. Fixations before skipped words could plausibly originate in such easy segments, and fixations before nonskipped words in normal or difficult segments. A second potentially confounding problem is that the durations of fixations depend on their locations in words. Fixations at the edges of a word are shorter than those occupying a middle position (Vitu, McConkie, Kerr, \& O'Regan, 2001). Fixations before skipping saccades are typically toward the ends of words. Therefore, we must also control for location within words. Third, we also need to control for interindividual differences in reading speed because fast reading is associated with a high skipping probability and short fixations. In the present article, we apply novel pattern-matching and resampling procedures to control for (1) local effects of text difficulty, (2) fixation positions within words, and (3) individual differences in reading speed.

This still leaves open the possibility that intraindividual differences (e.g., fluctuations of attention) generate differences in fixation durations before skipped and nonskipped words. For example, during attentional lapses readers may use short fixations and long saccades, generating a main effect of skipping on fixation duration. This influence is beyond our statistical control procedures, but this alternative to the theoretical interpretation of skipping effects would lose most of its force if the difference between fixations before skipped and before nonskipped words depended on the properties of the next word (e.g., its length, frequency, or predictability).

Finally, in contrast to fixations before skipped words, there are consistent reports of longer fixations after them. The main explanation of this effect follows from the solidly established preview benefit (see, e.g., Rayner, 1998, for a review): A preview of the word to the right reduces fixation duration on this word when the eyes move there with the next saccade. Obviously, a word fixated after a skipping saccade received less or no preview on the previous fixation. We will use this condition as a validity check of our data selection.

\section{METHOD}

\section{Participants}

Thirty-three young ( $M=22$ years, range $=19-28$ years $)$ and 32 older adults $(M=70$ years, range $=65-83$ years $)$ reported in Kliegl, Grabner, Rolfs, and Engbert (2004) participated; these participants were pooled into one group, which was supplemented with an additional 115 participants ranging in age from 16 to 80 years. Moreover, we included data from a group of students reading with a reduced monitor contrast $(n=30)$ and a group of older adults who had been exposed to the sentences 3-6 months earlier $(n=$ 20). The participants received study credit or were paid $€ 5$.

\section{Apparatus, Materials, and Procedure}

Details of the materials and experimental procedure were described in Kliegl, Grabner, Rolfs, \& Engbert (2004). The participants read 144 sentences of the Potsdam corpus, comprising 1,138 words. Not counting the first word of each sentence, the frequencies of the two- to nineletter words ranged from 54 to 222 occurrences in the corpus; 78 words were longer than nine letters. CELEX frequency norms (Baayen, Piepenbrock, \& van Rijn, 1993) as well as predictabilities of each word given the previous words of the sentence, are available for all words. Four groups were tested with EyeLink I (250-Hz) systems, and one group was tested with EyeLink II (500-Hz) systems.

Absolute letter positions (LPs) of fixations within words were rescaled to fixation zones (FZs; range, 0-5) independent of word length (WL) with the formula $\mathrm{FZ}=$ floor $\{[(\mathrm{LP}-0.5) / \mathrm{WL}] * 5\}+1$ (see Vitu et al., 2001, p. 3519); floor rounds its argument toward the next lower integer.

\section{Pattern Matching and Resampling Statistics}

We located three-word segments in sentences that were read with one fixation per word in a forward direction (see Figure 1, top panel). Next, we identified four-word segments that differed from the three-word segments only by the skipping of word $n+1$ (middle panel) or the skipping of word $n-1$ (bottom panel). Note that the same word can be in more than one segment for a given individual. Triplet pattern matching is illustrated by the black border in Figure 1; the word triplet indicates that three words are involved, each fixated once in a forward sequence. Sequences were matched on word $n$, and fixation zone was matched within word $n$. There are three types of matches: (1) nonskip and before-skip patterns, (2) nonskip and after-skip patterns, and (3) matches across all three patterns. Word $n$ was never the target of a regressive saccade.

To test the difference between fixation durations on word $n$ between patterns, we ran the following three-step procedure 100 times, yielding 100 repeated samplings. First, we randomly assigned each participant to exactly one of the patterns under comparison. For example, in a given sample a participant could contribute either to the nonskip pattern or to the before-skip pattern, but not to both. Thus, across the 100 samples, the participants contributed approximately equally often to the pools of nonskip and skip patterns, effectively controlling for individual differences in the frequencies of nonskip and skip patterns and the associated differences in reading speed.

Second, we selected a set of unique matching patterns from these pools. A unique pattern is jointly defined by word $n$ 's absolute position in the corpus and its fixation zone. Each pattern could appear only once in a given sample; if the pool contained several instances of a pattern (i.e., if 2 or more participants contributed the same pattern to one of the two pools), one was selected at random. Thus, in a given sample we did not use all instances of matching patterns but 


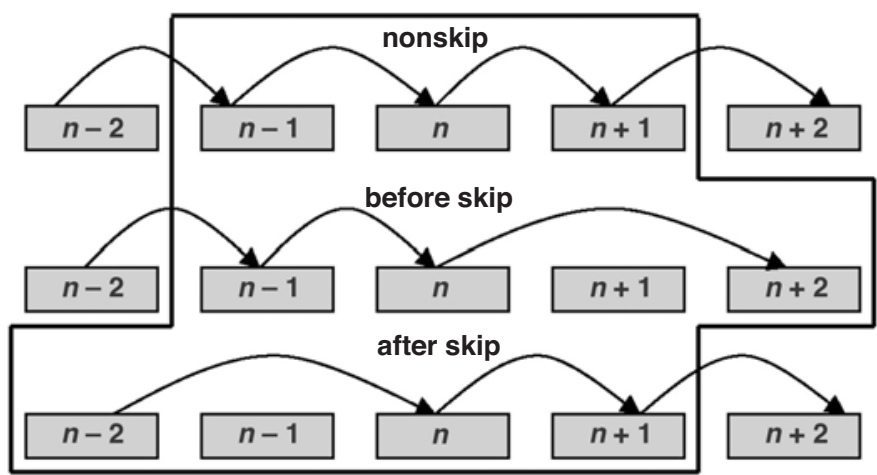

Figure 1. Three reading patterns for comparing fixation durations on word $\boldsymbol{n}$ among a nonskip pattern (top), a before-skip pattern (middle), and an after-skip pattern (bottom) in the same five-word segment of a sentence. Relative fixation positions within words were matched only for word $n$. The black border delineates triplet patterns used in the analyses.

only a unique set of them. However, across the 100 samples, all instances of a pattern had the same chance of being selected.

Third, we computed test statistics for the differences of fixation durations on matched elements (i.e., word + fixation zone) from the 100 samples. The standard deviations of the 100 sample means for each pattern and of the differences between matched means yield estimates of associated standard errors ( $S E \mathrm{~s}$; see, e.g., Efron $\&$ Tibshirani, 1993). In addition, we required that more than 95 of the 100 samples have the same sign in the difference scores for statistical significance (i.e., ., $\alpha=.05$ ).

\section{RESULTS}

The database contains 121,321 words with a single fixation that lasted $30-1,000 \mathrm{msec}$. The mean fixation duration was $200 \mathrm{msec}(S D=70 \mathrm{msec})$ and was located in the middle zone of the words $(M=2.6, S D=1.7)$. Of these fixations, 28,873 occurred before and 24,760 occurred after a skipping saccade. For a subset of these readers, Kliegl et al. (2004) reported independent effects of word length, frequency, and predictability on inspection durations (i.e., gaze, single, and first-fixation durations) as well as inspection probabilities for skipping, single fixations, and multiple fixations with repeated measures regression analyses. This basic pattern of results replicated in the pattern of results found for additional groups tested since then (i.e., a group varying across the adult age span, a group reading with reduced contrast, and a group reading with long-term prior exposure to sentences, respectively).

Means and standard deviations of durations and locations of fixations within words for the database used in this study are listed in Table 1 (top block), along with statistics for single fixations that were neither preceded nor followed by a skipping saccade. Overall, fixations were slightly shorter before and slightly longer after word-skipping saccades (195 and $204 \mathrm{msec}$, respectively) than the mean of other single fixations $(201 \mathrm{msec})$. Fixations before skips were toward the end of words (relative fixation zone $=2.9$ ), and those after skips were toward the beginning of words (1.6) relative to the location of nonskip fixations (2.5).

\section{Matching Word Triplets}

The matching analyses yielded similar results. In the second block of Table 1, the data for successively fixated word triplets are listed. In the "unmatched" section of this block, statistics for the nonskip, before-skip, and after-skip patterns (i.e., the patterns delineated by the black border in Figure 1) are reported. The "matched before" section contains statistics for when word $n$ was matched for nonskip and before-skip patterns; similarly the "matched after" section refers to data from the matching of nonskip and after-skip patterns. Finally, the "matched before and after" statistics are based on the matching of all three patterns on the identity and fixation zone of word $n$. Skipping effects were $-3 \mathrm{msec}$ (before skip - nonskip) and a statistically reliable $+17 \mathrm{msec}$ (after skip - nonskip) for pairwise matches, and $-4 \mathrm{msec}$ and $+20 \mathrm{msec}$ for the triple match for fixations before and after skipping saccades, respectively. The average number of repeatedly sampled matched patterns decreased sharply, from 1,735 and 1,332 for fixations before and after skipping saccades, respectively, to 50 , because for each pattern match 3 participants were needed who fixated word $n$ in the same fixation zone and generated the nonskip, beforeskip, and after-skip sequences.

Fixation locations followed the expected patterns. As is shown in Table 1, unmatched fixations before a skipping saccade occurred at the end of words, whereas fixations following a skip were toward their beginnings. Nonskip fixations tended to be located in the center of words. Therefore, matching pulled fixations toward the middle zone of words; fixation location no longer contributed to skipping effects on fixation durations. ${ }^{1}$

\section{Length, Frequency, and Predictability Effects of Skipped/Next Words on Fixation Durations}

The overall results look similar to those of previous analyses of eye movement corpora (McConkie et al., 1994; Radach \& Heller, 2000). Given the size of the present database, however, we could also check for the 
Table 1

Mean Durations (in Milliseconds) and Mean Locations (in Words) of Fixations Before and After Word-Skipping Saccades for Unmatched Single Fixations and for Unmatched and Matched Fixations in Word Triplets

\begin{tabular}{|c|c|c|c|c|c|c|c|}
\hline \multirow[b]{2}{*}{ Fixation } & \multirow[b]{2}{*}{$N$} & \multirow[b]{2}{*}{$\% \mathrm{CD}$} & \multicolumn{3}{|c|}{ Duration (msec) } & \multicolumn{2}{|c|}{$\begin{array}{l}\text { Location (Relative } \\
\text { Fixation Zone) }\end{array}$} \\
\hline & & & $M$ & $S D$ & $S E$ & $M$ & $S D$ \\
\hline \multicolumn{8}{|l|}{ Single fixations, unmatched } \\
\hline Nonskip & 72,462 & & 201 & 72 & & 2.5 & 1.4 \\
\hline Before & 28,873 & & 195 & 68 & & 2.9 & 1.5 \\
\hline After & 24,760 & & 204 & 65 & & 1.6 & 1.4 \\
\hline \multicolumn{8}{|l|}{ Fixation triplets } \\
\hline \multicolumn{8}{|l|}{ Unmatched } \\
\hline Nonskip & 53,719 & & 204 & 70 & & 2.5 & 1.3 \\
\hline Before & 17,778 & & 198 & 66 & & 3.2 & 1.3 \\
\hline After & 13,592 & & 211 & 65 & & 1.5 & 1.4 \\
\hline Matched before & 1,735 & 71 & & & & & \\
\hline Nonskip & & & 204 & & 3 & 2.9 & \\
\hline Before & & & 201 & & 3 & 2.9 & \\
\hline After & & & - & & - & - & \\
\hline Matched after & 1,332 & 100 & & & & & \\
\hline Nonskip & & & 201 & & 3 & 2.0 & \\
\hline Before & & & - & & - & - & \\
\hline After & & & $218^{*}$ & & 2 & 2.0 & \\
\hline Matched before and after & 50 & 59 (before) & & & & & \\
\hline Nonskip & & & 204 & & 9 & 2.3 & \\
\hline Before & & & 200 & & 10 & 2.3 & \\
\hline After & & & 224 & & 9 & 2.3 & \\
\hline
\end{tabular}

Note-Matched before, next word skipped; matched after, previous word skipped; \%CD, consistent direction of difference across 100 samples; $S E$, standard deviation of resampled means. Ns for the "matched" sections give the mean sizes of 100 samples from the common pool of triplets. $* p<.05$ (i.e., fewer than 5 of 100 differences in opposite directions; see $\% \mathrm{CD}$ ).

dependency of the skipping effect on the length, frequency, and predictability of the next word. We contrasted before-skip and nonskip fixations from matched three-word fixation sequences.

A sufficient number of skipping patterns was available for word lengths of two to nine letters, with seven-, eight-, and nine-letter words combined. Fixations before twoletter words were shorter (i.e., $-11 \mathrm{msec}$; negative difference in 96 of 100 samples, $p<.05$ ) when words were skipped than when they were fixated (Figure 2, panel A). The opposite effect occurred for long next words: Fixations before skipped words were longer $(+21 \mathrm{msec})$ for seven- to nine-letter words (positive difference in 99 of 100 samples, $p<.05$ ). Differences for intermediate word lengths were $-7,-5,+1$, and $+5 \mathrm{msec}$ for threeto six-letter words, respectively. Thus, we observed negative, zero, and positive effects of skipping depending on the length of the upcoming word. The inset in panel A of Figure 2 displays this increase of the skipping effect per letter. The significant mean difference in slopes between before-skip and nonskip trends was $+5 \mathrm{msec}(S E=$ $2 \mathrm{msec}$ ). Fixations before skipping significantly increased with the length of the skipped word $(+3 \mathrm{msec}, S E=$ $1 \mathrm{msec}$ ).

The skipping effect was also observed for logarithmic word frequency classes (see Figure 2, panel B), with differences of $+14,+10,+3,-11$, and $-7 \mathrm{msec}$. Statistical differences were reliable for the $-11-\mathrm{msec}$ effect in the second highest frequency class $(S E=5 \mathrm{msec}$, negative difference in 99 of 100 samples) and marginally significant for the positive effect associated with the +14 msec effect in the lowest frequency class $(S E=9 \mathrm{msec}$, positive difference in 91 of 100 samples; see inset in middle panel of Figure 2). The significant mean difference in slopes between before-skip and nonskip trends was $-8 \mathrm{msec}(S E=3 \mathrm{msec})$. Fixations before skipping significantly decreased with the logarithmic frequency of the skipped word $(-5 \mathrm{msec}, S E=2 \mathrm{msec}){ }^{2}$

Finally, there were no statistically reliable effects associated with logit predictability classes of the upcoming word, plotted in the panel $\mathrm{C}$ of Figure 2. This was not due to low reliability of the measure, because predictability is significantly related to durations on fixated words (Kliegl et al., 2004). In summary, the change of the skipping effect from negative to positive with increases in word length and decreases in word frequency likely explains the diverse set of results from previous research.

\section{Effects for Target Words Uncorrelated in Length and Frequency}

The overall correlation between word length and word frequency is -.59 for words of the Potsdam corpus. However, by design each sentence contained one target noun or verb for which length and frequency were uncorrelated across sentences, comprising a 6 (length) $\times 2$ (frequency) design with 12 words in each cell. For words with three 

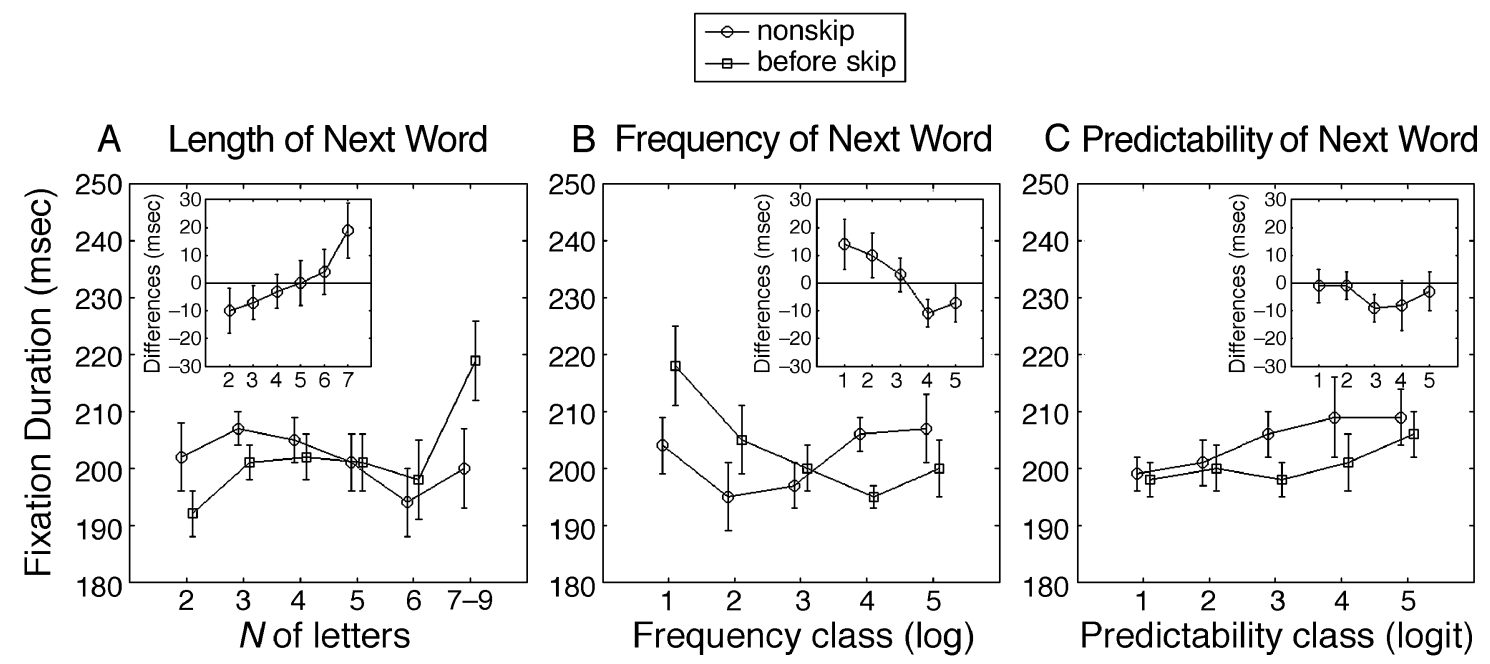

Figure 2. Fixation durations (in milliseconds) before skipped words and before triplet-matched nonskipped words as a function of (A) the length of the next word, (B) next-word frequency class (log), and (C) next-word predictability class (logit). Each error bar $( \pm 1 S E)$ is the standard deviation of 100 resampled means. Insets: Differences, in milliseconds $( \pm 1 S E)$ between fixation durations (i.e., before-skip - nonskip).

to five letters, skipping rate was sufficiently high to warrant statistical analyses of triplet-matched sequences. The number of patterns available for the resampling analyses illustrates the strong relation between skipping and frequency (i.e., $N=117$ for low frequency vs. 1,088 for high frequency) and between skipping and length (i.e., $N=711,234$, and 132 for words with three, four, and five letters, respectively). Means and $S E$ s from the resampling analyses are presented in Table 2.

Most important, there was a clear trend for main effects of word length and word frequency on fixations before these words when they were skipped. Relative to the nonskip pattern, fixations were $8 \mathrm{msec}$ longer before low-frequency target words were skipped and $7 \mathrm{msec}$ shorter before high-frequency target words were skipped; this 15 -msec difference of differences was marginably reliable $(t=1.34, p<.10)$. Similarly, for both low- and high-frequency words the difference between beforeskip and nonskip patterns changed toward increasingly positive values as word length increased from three to five letters (i.e., $-5.5 \mathrm{msec},+2.5 \mathrm{msec}$, and $+7.5 \mathrm{msec}$, respectively). Inspection of Table 2 shows that this trend was more pronounced for low-frequency words, but the statistical power was not sufficient to secure the significance of main effect and interaction related to this restricted range of word lengths. Nevertheless, it appears that both frequency and length of skipped words influenced fixation durations on the previous word.

\section{DISCUSSION}

The main conclusion from the present set of analyses is that fixations are shorter before skipped short and high-frequency words and significantly longer before

Table 2

Mean Pattern-Matched Fixation Durations (in Milliseconds) Before Skipped and Nonskipped Target Words Uncorrelated in Length and Frequency

\begin{tabular}{|c|c|c|c|c|c|c|c|c|c|c|c|c|}
\hline \multirow[b]{3}{*}{ Target Words } & \multicolumn{9}{|c|}{ Word Length } & & & \\
\hline & \multicolumn{3}{|c|}{3 Letters } & \multicolumn{3}{|c|}{4 Letters } & \multicolumn{3}{|c|}{5 Letters } & \multicolumn{3}{|c|}{ Total } \\
\hline & $M$ & $S E$ & $N$ & $M$ & $S E$ & $N$ & $M$ & $S E$ & $N$ & $M$ & $S E$ & $N$ \\
\hline Low frequency* & & & 28 & & & 39 & & & 29 & & & 117 \\
\hline Skipped & 200 & 12 & & 224 & 11 & & 223 & 14 & & 216 & 6 & \\
\hline Nonskipped & 197 & 12 & & 214 & 8 & & 207 & 16 & & 208 & 7 & \\
\hline$\Delta$ & 3 & 16 & & 10 & 15 & & 16 & 19 & & 8 & 10 & \\
\hline High Frequency** & & & 711 & & & 234 & & & 132 & & & 1,088 \\
\hline Skipped & 199 & 3 & & 198 & 4 & & 196 & 5 & & 198 & 3 & \\
\hline Nonskipped & 207 & 4 & & 204 & 5 & & 197 & 6 & & 205 & 3 & \\
\hline$\Delta$ & -8 & 5 & & -5 & 7 & & -1 & 8 & & -7 & 5 & \\
\hline
\end{tabular}

Note- $M, S E$, and $N=$ mean, standard deviation of resampled means, and average number of matched patterns for 100 samples, respectively. ${ }^{*}$ There were 36 low-frequency words; mean frequency $=2$ per million, maximum frequency $=4$ per million. ${ }^{* *}$ There were 35 high-frequency words; mean frequency $=801$ per million, minimum frequency $=46$ per million. 
skipped long and low-frequency words in comparison with fixations on the same words when they were followed by fixations on the next word (i.e., nonskipping patterns). The inconsistent picture emerging from earlier research could easily be a consequence of this tradeoff. Indeed, when we left out length and frequency information in the first set of analyses, we also observed a tendency toward shorter fixations before skipping, in consistency with previous corpus analyses (McConkie et al., 1994; Radach \& Heller, 2000). We also replicated the finding that fixations are reliably longer on words following a word-skipping saccade, most likely linked to reduced preview benefits for these words. This effect was much clearer after statistical control via pattern matching than it was with the three unselected conditions (see Table 1). It was also obtained at all levels of frequency and word length.

The combination of pattern matching and resampling procedures implements a degree of statistical control not enforced in previous studies. Here, word characteristics could not contribute to the effects on fixation durations because exactly the same word sequences were used in each comparison. In addition, within-word fixation location (i.e., relative fixation zone) was equated in the matching analyses. Finally, in each sample the readers contributed only to skipping or only to nonskipping patterns. Since the assignment of readers to these patterns was random for each sample, we guarded against selective contributions resulting from differences in reading speed. This leaves intraindividual cognitive fluctuations as a potential source of differences between fixations before skipped and nonskipped words. It is unclear, however, how this account can be related to the systematic modulations of the skipping effect by word length or frequency. Obviously, parafoveal word properties can influence fixations on prior words.

One caveat appears to be in order: We analyzed the most frequent fixation sequences, but the analyses do not necessarily generalize to other reading patterns. Of course, they can be extended in this direction. For example, unmatched fixations occurring after a skipping saccade and before a regressive saccade to the skipped word were shorter than nonskip fixations (184 vs. $204 \mathrm{msec}$, respectively), similar to the finding of Brysbaert and Vitu (1998). This difference was substantially reduced with pattern matching and resampling (194 vs. $200 \mathrm{msec}$, respectively).

What are the implications of these results for computational models of reading? In E-Z Reader 7 (Reichle et al., 2003), skipping occurs if (1) the eyes fixate word $n$ and (2) attention completes the first stage of lexical access of word $n+1$ (Stage L1) and shifts to word $n+2$ while the eyes still fixate word $n$ with a saccade program intended for word $n+1$ in the labile stage. In this case, the current saccade program is replaced with a new one to word $n+2$ after completion of Stage L1 for word $n+1$. Overall, such a saccade program cancelation increases fixation duration on word $n$ (see Table 1 in Reichle et al., 2003). Obviously, E-Z Reader did not predict the ob- served modulation of fixation duration before skipped words. There are, however, two model mechanisms that might be brought to bear on this issue: In E-Z Reader 7, skipping can occur due to misguided saccades, and there are no skipping costs for saccades canceled in the first substage of the labile stage of saccade programs. An account of the pattern reported here, however, will require simulations of word length effects as well as of effects of fixation position within words.

Word frequency and word length effects on fixation durations before skipped words do not fare well with models emphasizing POC. Moreover, the extended optimal viewing position (EOVP) model, which places itself within this tradition (Drieghe et al., 2004), was banking on predictability, which appeared to be the weakest of the three predictors in the present study. Finally, in principle, GAG models such as SWIFT (Engbert et al., 2002) and Glenmore (Reilly \& Radach, 2003) can accommodate frequency and length effects on fixation durations before skipping, but the empirical signature reported here still needs to be reproduced in simulations. Moreover, in the SWIFT model, longer fixation durations before skipped words due to frequency and length can arise only as a result of the distributed parallel processing of words in the perceptual span, rather than as a result of saccade cancelation.

As a concluding side note, we mention that the effects of skipping discussed here underscore the importance of computational modeling, since it is close to impossible to generate specific and quantitative predictions without implementing a model on a computer. Since the latest of these models are very detailed and are based on a large set of parameters, many experimentalists believe that there is always a loophole through which complex models ably escape from falsification. We have demonstrated that sophisticated models or a subset of their underlying assumptions can be tested with correlational analyses of fixation patterns from reading of continuous text. Obviously, supplemental experimental evidence would be very desirable. It is to the credit of the E-Z Reader model that it stimulated the search for a resolution of empirical inconsistencies related to fixation durations before word skipping.

\section{REFERENCES}

BaAyen, R. H., Piepenbrock, R., \& van RiJn, H. (1993). The CELEX lexical database [CD-ROM]. Philadelphia: University of Pennsylvania, Linguistic Data Consortium.

BrysbaerT, M., \& VITU, F. (1998). Word skipping: Implications for theories of eye movement control in reading. In G. Underwood (Ed.), Eye guidance in reading and scene perception (pp. 125-147). Oxford: Elsevier.

Drieghe, D., Brysbaert, M., Desmet, T., \& De Baecke, C. (2004). Word skipping in reading: On the interplay of linguistic and visual factors. European Journal of Cognitive Psychology, 16, 79-103.

Efron, B., \& Tibshirani, R. J. (1993). An introduction to the bootstrap. New York: Chapman \& Hall.

Engbert, R., \& KLIEGL, R. (2001). Mathematical models of eye movements in reading: A possible role for autonomous saccades. Biological Cybernetics, 85, 77-87.

ENGBERT, R., LONGTIN, A., \& KLIEGL, R. (2002). A dynamical model 
of saccade generation in reading based on spatially distributed lexical processing. Vision Research, 42, 621-636.

Hogaboam, T. W. (1983). Reading patterns in eye movements. In $\mathrm{K}$. Rayner (Ed.), Eye movements in reading: Perceptual and language processes (pp. 309-332). New York: Academic Press.

KLIEGL, R., \& ENGbert, R. (2003). SWIFT explorations. In J. Hyönä, R. Radach, \& H. Deubel (Eds.), The mind's eye: Cognitive and applied aspects of eye movement research (pp. 391-411). Amsterdam: Elsevier.

Kliegl, R., Grabner, E., Rolfs, M., \& Engbert, R. (2004). Length, frequency, and predictability effects of words on eye movements in reading. European Journal of Cognitive Psychology, 16, 262-284.

McConkie, G. W., Kerr, P. W., \& Dyre, B. P. (1994). What are "normal" eye movements during reading: Toward a mathematical description. In J. Ygge \& G. Lennestrand (Eds.), Eye movements in reading (pp. 315-327). Oxford: Pergamon.

Pollatsek, A., RAYNer, K., \& Balota, D. A. (1986). Inferences about eye movement control from the perceptual span in reading. Perception \& Psychophysics, 40, 123-130.

PynTE, J., KennedY, A., \& DucRot, S. (2004). The influence of parafoveal typographical errors on eye movements in reading. European Journal of Cognitive Psychology, 16, 178-202.

RADACH, R., \& HELlER, D. (2000). Relations between spatial and temporal aspects of eye movement control. In A. Kennedy, R. Radach, D. Heller, \& J. Pynte (Eds.), Reading as a perceptual process (pp. 165192). Oxford: Elsevier.

RAYNER, K. (1998). Eye movements in reading and information processing: 20 years of research. Psychological Bulletin, 124, 372-422.

Reichle, E. D., Pollatsek, A., Fisher, D. L., \& Rayner, K. (1998).
Toward a model of eye movement control in reading. Psychological Review, 105, 125-157.

Reichle, E. D., RAYNer, K., \& Pollatsek, A. (2003). The E-Z Reader model of eye movement control in reading: Comparisons to other models. Behavioral \& Brain Sciences, 26, 445-526.

REILLY, R., \& RADACH, R. (2003). Foundations of interactive activation model of eye movement control in reading. In J. Hyönä, R. Radach, \& H. Deubel (Eds.), The mind's eye: Cognitive and applied aspects of eye movement research (pp. 429-456). Amsterdam: Elsevier.

Vitu, F., McConkie, G. W., Kerr, P., \& O'Regan, J. K. (2001). Fixation location effects on fixation durations during reading: An inverted optimal viewing position effect. Vision Research, 41, 35133533.

\section{NOTES}

1. Similar results were obtained for analyses of five-word segments (quintets) such as that illustrated in Figure 1. These analyses also controlled for the boundary conditions of the reading context (i.e., the first fixation in the segment was always on word $n-2$ and the last fixation on word $n+2$ ).

2. Estimates were based on 100 resampled matched pairs of multiple regressions, which also included dummy codes for between-subjects differences. All 100 differences between linear coefficients were positive for word length and negative for word frequency.

(Manuscript received October 1, 2003; revision accepted for publication March 9, 2004.) 